\title{
Initial review on ICTS governance for software anti-aging
}

\begin{abstract}
For the past 20 years various researches regarding software aging have been conducted. Software aging is the situation in which the accumulation of errors occurring in operational software system that has run for a long time that may lead to performance degradation, resource depletion and eventually causing the software to crash or hang [1]. David Parnas divided software aging into two categories: 1) the failure of the software to adapt with environment that is dynamic and 2) the result of the changes itself [2]. Factors that can affects software aging can be classified into several categories: 1) functional, 2) human, 3) product and 4) environment [3]. In general, the factors that affect software aging can be divided into internal and external factors. The main objectives of this paper are to briefly describe the definition of software aging and also ICTS governance. In addition to that, this paper also compiles the software aging factors that are being investigated by previous researchers. The need for future research regarding ICTS governance and Software aging also determined at the end of this paper.
\end{abstract}

Keyword: Governance; ICTS; Anti aging 